\title{
Tracking Control of a Nanopositioner Using Complementary Sensors
}

\author{
Iskandar A. Mahmood, S. O. Reza Moheimani, Senior Member, IEEE, and Kexiu Liu
}

\begin{abstract}
Piezoelectric tube actuators are widely used in atomic force and scanning tunneling microscopy (STM) for nanoscale positioning. There has been a consistent effort to increase the scan speed of these actuators using feedback control techniques. A feedback controller requires a measurement of the scanner's deflection, which is often provided by a capacitive sensor. Such measurements are corrupted by sensor noise, typically in the order of $20 \mathrm{pm} / \sqrt{\mathrm{Hz}} \mathrm{rms}$. Over a bandwidth of $10 \mathrm{kHz}$, this translates into an rms noise of $2 \mathrm{~nm}$, clearly inadequate for applications that require subnanometer positioning accuracy, e.g., STM. In this paper, we illustrate how the strain voltage induced in a free electrode of the scanner can be used as an additional displacement signal. The noise level corresponding to the strain signal is about three orders of magnitude less than that of a capacitive sensor, making it an ideal choice for nanopositioning applications. However, it cannot be used for dc and low-frequency measurements. A two-sensorbased controller is designed to use the capacitive sensor signal at low frequencies, and the strain displacement signal at high frequencies. By limiting the capacitive sensor feedback loop bandwidth to less than $100 \mathrm{~Hz}$, the rms value of the noise is reduced to well below $1 \mathrm{~nm}$. For almost the same noise level, the two-sensor-based control structure achieves a closed-loop bandwidth of more than three times that of the single-sensor-based controller.
\end{abstract}

Index Terms-Piezoelectric tube scanner, scanning probe microscopes (SPMs), two-sensor-based $\boldsymbol{H}_{\infty}$ controller.

\section{INTRODUCTION}

$\mathbf{P}$ IEZOELECTRIC transducers have been used in a wide range of applications such as vibration control of flexible structures [1]-[6], active noise control [7], [8], microelectromechanical systems (MEMSs) [9], and also in scanning probe microscopes (SPMs) [10]. The term SPM is a name given to a group of microscopes such as scanning tunneling microscope (STM), atomic force microscope (AFM), and similar devices that use a probe to develop images of material surfaces with extremely high accuracies.

Application of the piezoelectric tube was first reported in [11] for the use in the STM. In an SPM, the piezoelectric tube is used to either move the probe or the sample in a raster pattern in order to scan the entire region of interest. A raster scanning is normally performed by moving the piezoelectric tube along the $x$-axis in forward and reversed directions (horizontal scanning), and then moving the piezoelectric tube along the $y$-axis in a small step to reach the next line (frame scanning) for another

Manuscript received January 14, 2008; revised March 31, 2008. First published September 3, 2008; current version published January 16, 2009. This work was supported by the Australian Research Council. The review of this paper was arranged by Associate Editor B. Nelson.

The authors are with the School of Electrical Engineering and Computer Science, University of Newcastle, Callaghan, N.S.W. 2308, Australia (e-mail: iskandar.mahmood@newcastle.edu.au; reza.moheimani@newcastle.edu.au; kexiu.liu@newcastle.edu.au).

Digital Object Identifier 10.1109/TNANO.2008.2005183 horizontal scanning [12]. During the forward pass of horizontal scan, the surface topographic information gathered by the probe is stored for image processing. The speed of a piezoelectric scanner is largely determined by the scanning frequency of horizontal scanning.

The positioning precision of the piezoelectric tube can be adversely affected by vibrations and nonlinearities exhibited by the piezoelectric material such as hysteresis and creep [13]. The main source of vibrations is scan induced vibrations due to excitation of the lightly damped first resonant mode of the actuator by higher harmonics of the horizontal scanning signal. In practice, in order to minimize the induced vibrations, the horizontal scanning frequency is limited to $1 / 100$ th of the first resonance frequency of the tube, or lower. As for the nonlinearities, the adverse effects of hysteresis and creep can be reduced by limiting the scanning range and operating time of the piezoelectric tube, respectively. The aforementioned limitations restrict the use of the piezoelectric tube scanner for high speed and long scanning range operations.

There has been a consistent effort in recent years to improve accuracy and speed of scanning probe microscopes using feedback control techniques. One of the earliest attempts to control a piezoelectric tube actuator is reported in [14], where a noncontacting inductive sensor was used to measure the lateral displacement of a tube. Lag-lead and $H_{\infty}$ controllers were designed and implemented on the tube, and the feasibility of reducing the adverse effects of creep and hysteresis were demonstrated. The authors also reported that the $H_{\infty}$ controller achieved damping of high-frequency vibrations. The use of loop shaping procedure to design a feedback controller for a piezoelectric tube actuator instrumented with optical displacement sensors to track a raster pattern was described in [15]. Their results show reductions in tracking error and cross coupling due to the use of feedback control. Examples of other successful applications of feedback include [16]-[18]. A comprehensive review of the field can be found in [19].

The key idea associated with feedback-based methods is to damp the first resonant mode of the piezoelectric tube actuator. This "flattening" of the frequency response of the scanner will allow tracking of a faster triangular waveform, and consequently, a faster scan. Furthermore, to achieve accurate positioning at high frequencies and minimize the adverse effect of hysteresis, the feedback gain is often chosen to be high. There is a limit on how high the feedback gain can be made before the closed-loop system is made unstable, since the existence of sharp resonant peaks in the frequency response of the actuator typically results in a very low gain margin [20]. Using notch filters in the feedback loop has been shown to result in an improvement in the achievable gain margin [21]. 
The use of high-gain feedback for accurate tracking is necessitated due to the hysteretic nature of the piezoelectric actuator. If the actuator is driven by a charge source, the hysteresis is significantly reduced, resulting in an almost linear actuator, a fact that has been known since early 1980s [22], [23]. Recent progress in designing dc-accurate charge amplifiers has made it possible to use this unique property of piezoelectric materials [24]-[26]. A similar charge source is used here to actuate a prototype piezoelectric tube nanopositioner.

Inversion-based feedforward method has also been applied to piezoelectric tube actuators. An attractive feature of the feedforward control scheme is that this method does not require any additional sensors for implementation. Model-based inversion approach was used in [13] to compensate for positioning distortions caused by creep, hysteresis, and induced vibrations. A low-order feedforward controller was presented in [27] to suppress the lateral oscillation of a piezoelectric tube scanner. The feedforward controller was designed using $H_{\infty}$ method such that the system is not excited at frequencies around the first resonance of the piezoelectric tube scanner. The performance of feedforward control schemes heavily relies on an accurate model of the system [13], [27]. Combining feedback and feedforward compensations has been shown to result in satisfactory tracking in a piezoelectric tube nanopositioner in the presence of parameter uncertainties in the plant model [28], [29].

In applications where ultrahigh-precision positioning is a necessity, e.g., in scanning probe microscopy, the performance of the feedback control scheme is severely limited by the noise properties of the displacement sensor. To appreciate this, consider a displacement sensor that has an rms noise of $20 \mathrm{pm} / \sqrt{\mathrm{Hz}}-$ most capacitive and inductive displacement sensors are subject to this level of noise. If the sensor is operated over a bandwidth of, say $10 \mathrm{kHz}$, its rms noise will be $2 \mathrm{~nm}$, which makes it impossible to achieve subnanometer positioning accuracy. However, if the very same sensor is operated over a bandwidth of $100 \mathrm{~Hz}$, the noise level is reduced to $2 \AA$, about the radius of an atom. Limiting the bandwidth to $1 \mathrm{~Hz}$ would further reduce this noise level to $0.2 \AA$. Thus, the positioning accuracy achievable by a feedback controller can be significantly improved. However, this would also limit the operating bandwidth of the feedback controller, resulting in very slow closed-loop operation of the system. Such a severe closed-loop bandwidth limitation would come at the additional cost of making the closed-loop system sensitive to vibration, noise, and other disturbances.

The key idea of this paper is to utilize the piezoelectric voltage induced in one of the two electrodes as an additional displacement sensor. Although this signal cannot be used to measure static deflections of the tube, and it has a poor low-frequency response, it can function as an excellent high-frequency displacement sensor, with a noise level of at least three orders of magnitude less than a capacitive sensor. Thus, for all practical purposes, this sensor can be viewed to be almost free of noise. A controller can be designed to achieve satisfactory tracking using these two "complementary" sensors. Here, a two-input one-output $H_{\infty}$ controller is designed to use the capacitive sensor measurements at low frequencies (below $100 \mathrm{~Hz}$ ), and dc,

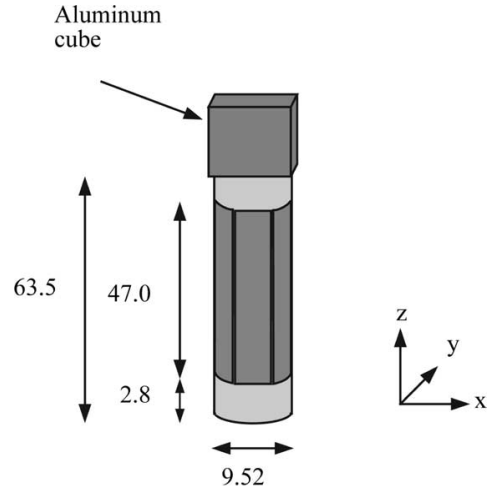

(a)

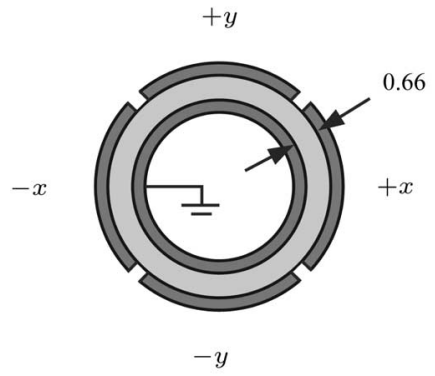

(b)

Fig. 1. Piezoelectric tube dimensions in millimeters. (a) Isometric view. (b) Bottom view (dimensions are not to the scale and the thickness of the electrode is exaggerated).

and the piezoelectric strain signal at higher frequencies. For roughly the same noise level, the controller achieves a closedloop bandwidth more than three times that obtained from a controller utilizing the capacitive sensor measurement alone.

The remainder of the paper is arranged as follows. Section II provides a description of the experimental setup. Modeling and identification of the system transfer functions are presented in Section III. Control schemes are devised in Section IV. In Section V, simulation and experimental results are presented to illustrate the effectiveness of the proposed control schemes. Finally, conclusions are drawn in Section VI.

\section{SYSTEM DESCRIPTION}

The piezoelectric tube used in the experiments reported in this paper is a cylindrical tube made of piezoelectric material plated with a layer of electrode on the inner and outer surfaces of the tube. The inner electrode is continuous and grounded. The outer layer electrode is segmented into four equal sized electrodes and referred individually as $+x,-x,+y$, and $-y$ electrodes. The physical dimensions of the tube are given in Fig. 1. The piezoelectric tube is housed in a circular aluminum enclosure to protect it from external disturbances and acoustic noise. A hollowed aluminum cube is glued to the top of the tube to serve as a sample holder and also to provide the capacitive sensors (ADE Technologies 2804 capacitive sensor) flat surfaces so that the tip deflection can be measured accurately. The capacitive sensors are fixed at right angles to the cube surface in the 


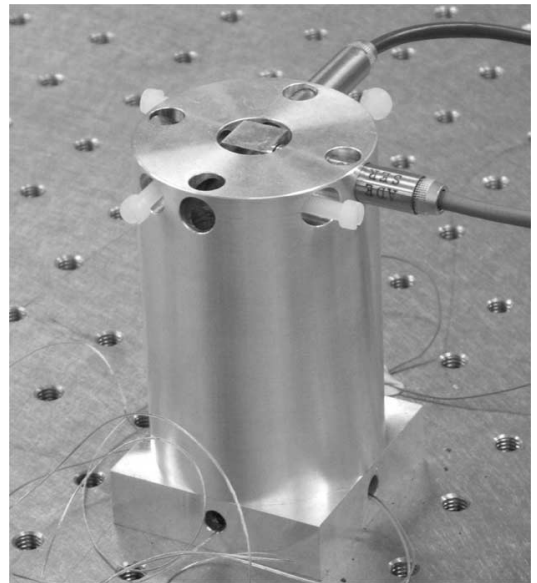

Fig. 2. Piezoelectric tube is housed in a circular aluminum enclosure.

$x$-axis and $y$-axis by using nylon screws, as shown in Fig. 2 . The capacitive sensors have a sensitivity of $10 \mu \mathrm{m} / \mathrm{V}$ over a range of $\pm 100 \mu \mathrm{m}$. The rms noise density of the capacitive sensors were measured in [30] to be $17.5 \mathrm{pm} / \sqrt{\mathrm{Hz}}$. Each of the capacitive sensors is driven by an ADE Technologies 4810 gaging system that comes with multiple bandwidth settings. By operating the sensor over a bandwidth of $100 \mathrm{~Hz}$, the rms noise or the resolution of the capacitive sensor is set to $0.175 \mathrm{~nm}$.

In most nanoscale positioning applications, including in most SPMs, the tube motion is produced by applying equal and opposite sign input signals to the electrodes opposite to each other. However, in this paper, only the $+x$ electrode is used to produce the forward and reversed motions of the tube in the $x$-axis. This is achieved by applying a triangular signal to this electrode. The opposite electrode $-x$ is used as a secondary sensor to measure the tip deflection. Note that this arrangement reduces the scan range of the tube to half. However, it results in a substantially higher positioning accuracy, as articulated shortly. If needed, a larger scan range can be obtained by utilizing a tube of different dimensions.

When the tube deflects, the piezoelectric strain voltage induced in the $-x$ electrode is found to be proportional to the tip deflection over a certain frequency range. The transfer function from the strain voltage to the output of an instrumentation amplifier resembles a first-order high-pass filter [3]. This is due to the capacitive nature of the piezoelectric tube. The high-pass filter can be expressed as

$$
G_{\mathrm{hp}}(s)=\frac{s}{s+\left(1 / R_{\mathrm{in}} C_{p}\right)}
$$

where $R_{\text {in }}$ is the input impedance of the voltage measuring instrument and $C_{p}$ is the capacitance of the piezoelectric tube.

The rms noise density of the piezoelectric strain voltage was measured in [30] and [31] to be $16 \mathrm{fm} / \sqrt{\mathrm{Hz}}$, about a thousand times less than that of the capacitive sensor. Such an extremely low-noise level will only cause a few picometers of rms noise over a bandwidth of tens of kilohertz. By this measure, this should be the preferred displacement sensor. However, due to its high-pass nature, as articulated before, accurate positioning at low frequencies using this sensor alone is impossible. At

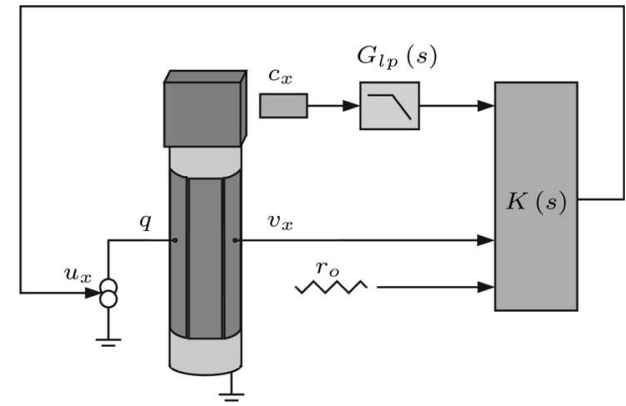

Fig. 3. Schematics of the proposed feedback control system.

low frequencies where the strain signal cannot be used, the displacement measurement obtained from the capacitive sensor can be used directly. The complementary nature of the two measurements allows for the bandwidth of the capacitive sensor to be made very low, thus reducing the overall effect of noise on the controlled position of the scanner to an absolute minimum.

The schematics of the proposed feedback control scheme are illustrated in Fig. 3. The $+x$ electrode is being driven by a home-made charge source [24], [25] that renders the plant linear, hence reducing the adverse effect of hysteresis. The charge amplifier has a constant gain of $68 \mathrm{nC} / \mathrm{V}$. The two-input oneoutput controller is designed to take advantage of the complementary nature of the two measurement signals. Details of the design are explained in Section IV. A dSPACE DS1103 controller board equipped with a 16-bit analog-to-digital converter (ADC)/digital-to-analog converter (DAC) cards was used for real-time controller implementation. A sampling frequency of $15 \mathrm{kHz}$ is used to avoid aliasing. In order to reduce the quantization noise, a low-noise preamplifier with a gain of 10 is used to amplify the capacitive sensor output so that it occupies the full range of the ADC card for a range of $\pm 10 \mu \mathrm{m}$.

\section{SYSTEM IDENTIFICATION}

This section discusses and details the modeling procedures undertaken in this paper. The following frequency response functions (FRFs) were determined using a dual channel HP35670A spectrum analyzer

$$
G_{v_{x} u_{x}}(i \omega)=\frac{v_{x}(i \omega)}{u_{x}(i \omega)}
$$

and

$$
G_{c_{x} u_{x}}(i \omega)=\frac{c_{x}(i \omega)}{u_{x}(i \omega)}
$$

where $u_{x}$ is the input voltage to the charge amplifier, $v_{x}$ is the induced piezoelectric strain voltage, and $c_{x}$ is the output voltage of the capacitive sensor. The subscript $x$ denotes that the actuation and measurements were performed along the $x$ axis. A band-limited random noise signal $(1-1601 \mathrm{~Hz})$ was generated using the spectrum analyzer and applied to the charge amplifier as the input $u_{x}$. The corresponding outputs $v_{x}$ and $c_{x}$ were also recorded using the same device. The input-output data were processed to generate the FRFs of (2) and (3) in a nonparametric form, as illustrated in Fig. 4. 

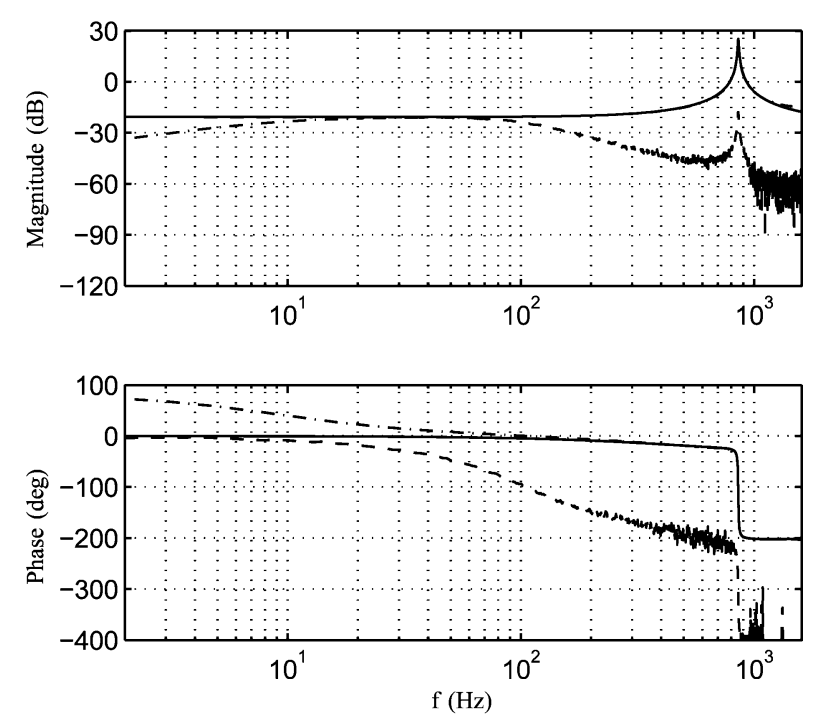

Fig. 4. One-loop-frequency responses, $G_{v_{x} u_{x}}(i \omega)$ (dash dots), $G_{c_{x} u_{x}}(i \omega)$ (dash), and the identified model $G_{y_{x} u_{x}}(s)$ (solid).

It can be seen from Fig. 4 that $G_{v_{x} u_{x}}(i \omega)$ includes a high-pass filter with a cutoff frequency of about $9 \mathrm{~Hz}$. The high-pass filter results in a phase lead and heavy attenuation of the strain voltage signal at low frequencies. Thus, the strain voltage cannot be used as a reliable tip deflection measurement at low frequencies. Nevertheless, from about $20 \mathrm{~Hz}$ and onwards, the strain voltage provides an excellent signal that can be used to estimate the tip deflection and also the dynamics of the piezoelectric tube. As for $G_{c_{x} u_{x}}(i \omega)$, its frequency response includes a low-pass filter with a cutoff frequency of $100 \mathrm{~Hz}$. Thus, the capacitive sensor can be used to measure the tip deflection below $70 \mathrm{~Hz}$ with acceptable accuracy. After this frequency, $G_{c_{x} u_{x}}(i \omega)$ starts to roll off considerably. These two measurements complement each other since the former is accurate at high frequencies, including at the resonance, and the latter is reliable at low frequencies, including at dc. Note that a scaling factor of $K_{a}=0.2$ has been

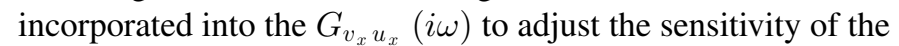
signal obtained from $-x$ electrode and make it identical to that of the capacitive sensor signal.

In this paper, instead of fitting separate transfer functions to $G_{v_{x} u_{x}}(i \omega)$ and $G_{c_{x} u_{x}}(i \omega)$, a new FRF was formed by using the low-frequency range $(1-50 \mathrm{~Hz})$ of the $G_{c_{x} u_{x}}(i \omega)$ data and the high-frequency range $(51-1601 \mathrm{~Hz})$ of the $G_{v_{x} u_{x}}(i \omega)$ data. The new FRF corresponds to the deflection of the tip $y_{x}$ that is not affected by artifacts such as the high-pass property of the strain signal or the low-pass property of capacitor sensor measurement. A second-order model was fitted to the new FRF data. The identification algorithm used for this purpose was the frequency-domain subspace-based system identification approach described in [32] and [33]. The following model was found to be a good fit, as illustrated in Fig. 4:

$$
G_{y_{x} u_{x}}(s)=\frac{-0.06 s^{2}-342.8 s+2.654 \times 10^{6}}{s^{2}+49.47 s+2.895 \times 10^{7}} .
$$

The high-pass and the low-pass filter characteristics corresponding to $G_{v_{x} u_{x}}(i \omega)$ and $G_{c_{x} u_{x}}(i \omega)$, respectively, are fitted

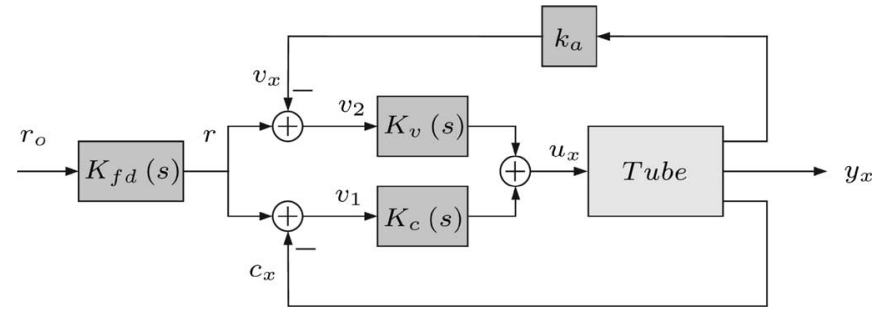

Fig. 5. 2-DOF control block diagram.

with the following models:

$$
G_{\mathrm{lp}}(s)=\frac{3.948 \times 10^{5}}{s^{2}+888.6 s+3.948 \times 10^{5}}
$$

and

$$
G_{\mathrm{hp}}(s)=\frac{s}{s+55.29} .
$$

\section{Controller Design}

This section discusses and details the $H_{\infty}$ control design schemes proposed in this paper. The key objectives of the design are as follows.

1) To achieve good damping ratio for the first resonant mode of the piezoelectric tube scanner.

2) To achieve higher tracking bandwidth using the lowbandwidth capacitive sensor and the piezoelectric strain voltage signal as the primary and secondary displacement measurements, respectively.

3) To minimize the effect of low-frequency vibrations on the tube's deflection.

For the sake of comparison, a second controller using only the measurements obtained from the capacitive sensor is also designed to achieve the aforementioned objectives as much as possible.

\section{A. Two-Sensor-Based $H_{\infty}$ Controller}

The proposed control diagram is illustrated in Fig. 5, where a 2-DOF controller scheme is to be synthesized. The control structure consists of the feedforward controller $K_{\mathrm{fd}}(s)$ and the feedback controllers $K_{v}(s)$ and $K_{c}(s)$. The feedback controllers are first designed. Fig. 6 illustrates the feedback control block diagram with incorporated weighting functions. The problem can be cast into the standard $H_{\infty}$ controller design framework, as shown in Fig. 7. The exogenous input vector is defined as

$$
w=\left[\begin{array}{c}
r \\
d_{i} \\
n
\end{array}\right]
$$

and the exogenous output as

$$
z=\left[\begin{array}{l}
z_{1} \\
z_{2}
\end{array}\right]
$$

where $r$ is the reference signal to be tracked, $d_{i}$ represents low-frequency vibrations modeled as an input disturbance, and $n$ represents the sensor noise. Furthermore, $u_{x}$ is the control 


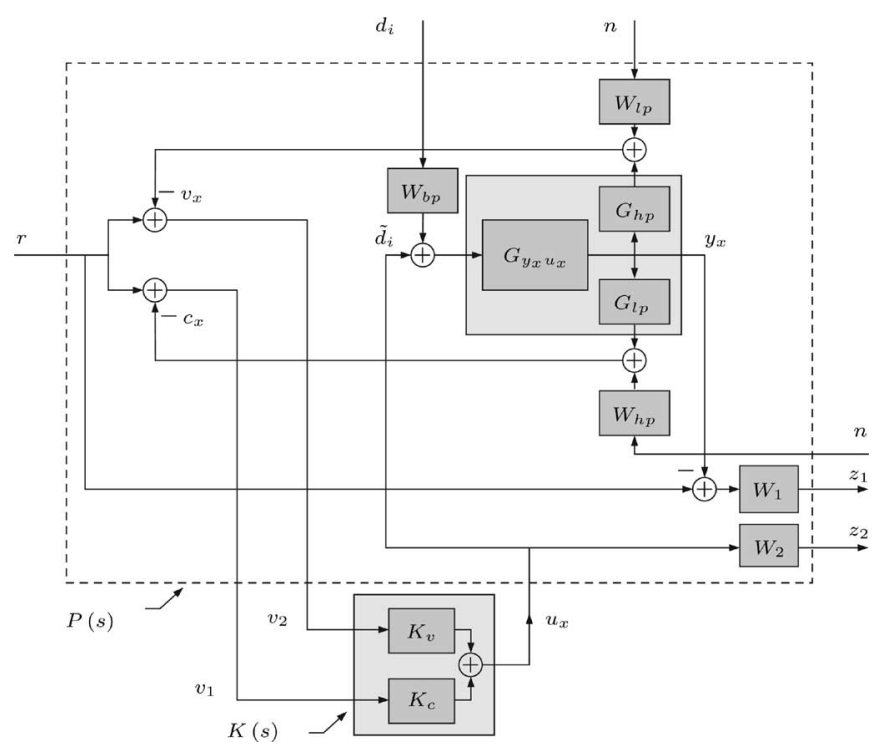

Fig. 6. Feedback control block diagram with weighting functions.

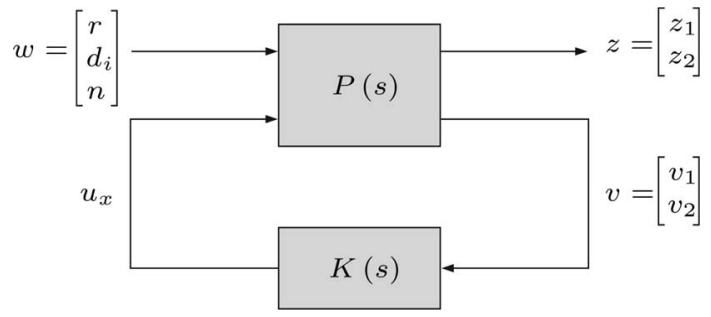

Fig. 7. General feedback control configuration.

signal and $v$ is the measured output. From Fig. 6, it is clear that

$$
z_{1}=W_{1}\left(r-G_{y_{x} u_{x}}\left(u_{x}+W_{\mathrm{bp}} d_{i}\right)\right)
$$

and

$$
z_{2}=W_{2} u_{x}
$$

The control signal is $u_{x}$ and $v$ is the vector of measured outputs

$$
v=\left[\begin{array}{l}
v_{1} \\
v_{2}
\end{array}\right]
$$

where

$$
v_{1}=r-\left(G_{y_{x} u_{x}}\left(u_{x}+W_{\mathrm{bp}} d_{i}\right) G_{\mathrm{lp}}+W_{\mathrm{hp}} n\right)
$$

and

$$
v_{2}=r-\left(G_{y_{x} u_{x}}\left(u+W_{\mathrm{bp}} d_{i}\right) G_{\mathrm{hp}}+W_{\mathrm{lp}} n\right) .
$$

To achieve satisfactory vibration reduction at low frequencies, a disturbance $d_{i}$ has been introduced at the input of the plant and the controller is forced to minimize $T_{y_{x} d_{i}}$, the transfer function from the input disturbance $d_{i}$ to the actual nanopositioner output $y_{x}$. The weighting function $W_{\mathrm{bp}}$ is tuned to the resonance frequency of the tube that is located in the vicinity of $850 \mathrm{~Hz}$, as shown in Fig. 4, and is chosen as

$$
W_{\mathrm{bp}}(s)=\frac{1793 s}{s^{2}+1793 s+2.893 \times 10^{7}} .
$$

and

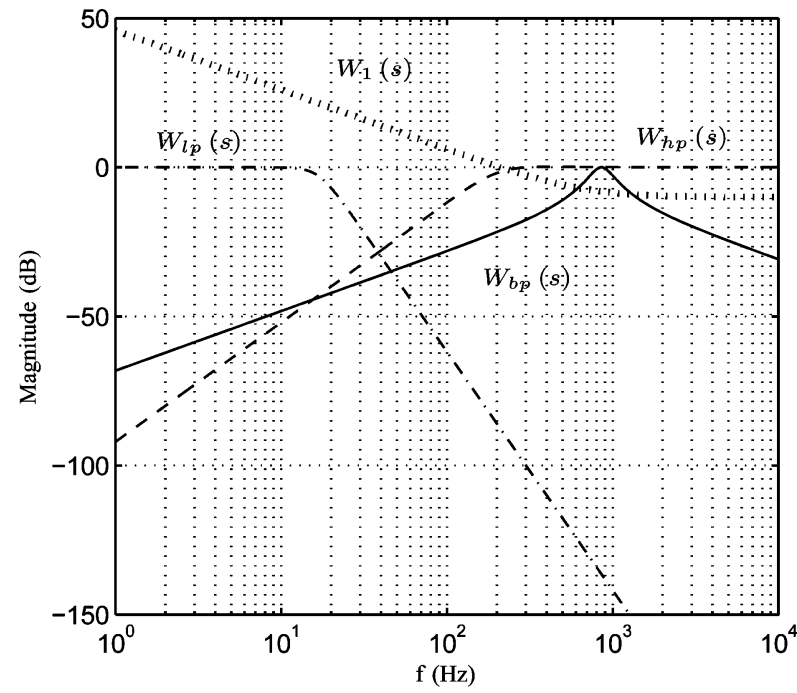

Fig. 8. Weighting functions.

The capacitive sensor is noisy at high frequencies, and the piezoelectric stain voltage signal is distorted at low frequencies. The controller is designed to utilize the capacitive sensor for tracking low-frequency signals, and the strain voltage for tracking signals that contain higher frequency components. This is achieved by introducing the two weighting functions $W_{\mathrm{hp}}$ and $W_{\mathrm{lp}}$, as shown in Fig. 6, as

$$
\begin{aligned}
W_{\mathrm{lp}}(s)= & \frac{1.262 \times 10^{8}}{\left(s^{2}+195.9 s+1.124 \times 10^{4}\right)} \\
& \times \frac{1}{\left(s^{2}+81.13 s+1.124 \times 10^{4}\right)}
\end{aligned}
$$

$$
W_{\mathrm{hp}}(s)=\frac{s^{2}}{s^{2}+1571 s+1.579 \times 10^{6}} .
$$

Furthermore, $W_{2}=0.1$ is used to impose a constraint on the control signal. This is to avoid excessively large control signals that could saturate the actuator.

The weighting function $W_{1}$ is incorporated to enforce good tracking performance. The inverse of this transfer function can be considered as the desired sensitivity transfer function $T_{e r}$, the transfer function from reference signal $r$ to the tracking error $e=r-y_{x} . W_{1}$ is chosen as

$$
W_{1}(s)=\frac{0.3162 s+1257}{s+1.257} .
$$

Fig. 8 illustrates the main weighting functions. To examine the effectiveness of the controller, the achieved sensitivity function $S(s)$ is plotted against the desired sensitivity function $W_{1}^{-1}(s)$ in Fig. 9. The figure illustrates that both of the achieved and the desired sensitivity functions match closely except at high frequencies, particularly beyond $1 \mathrm{kHz}$. This indicates that the synthesized two-input one-output $H_{\infty}$ controller performs as intended.

Fig. 10 plots the frequency responses of the feedback controllers $K_{c}(s)$ and $K_{v}(s)$. It can be observed that the two 


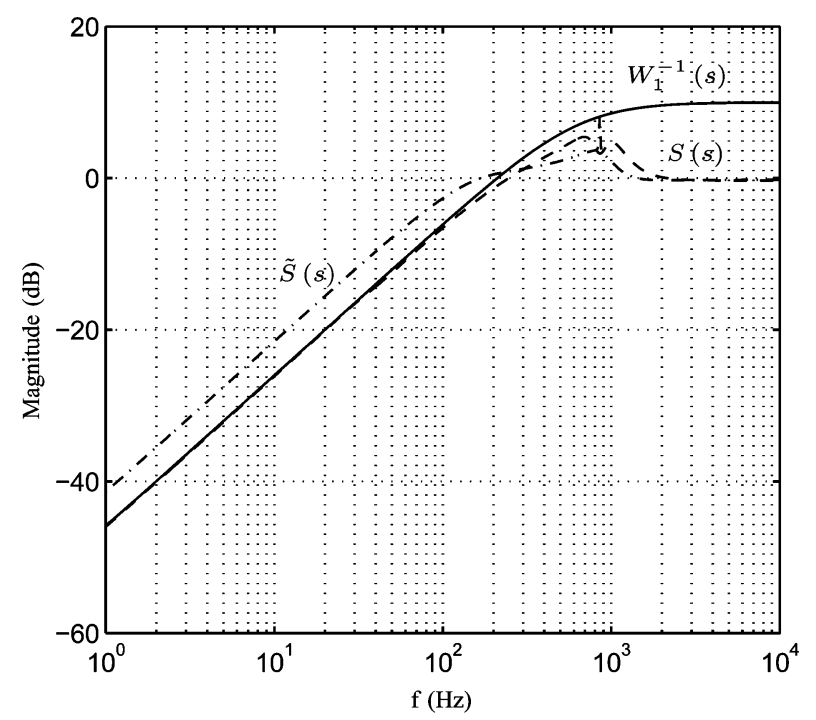

Fig. 9. Sensitivity functions: desired (solid), achieved two sensor (dash), and achieved single sensor (dash dot).

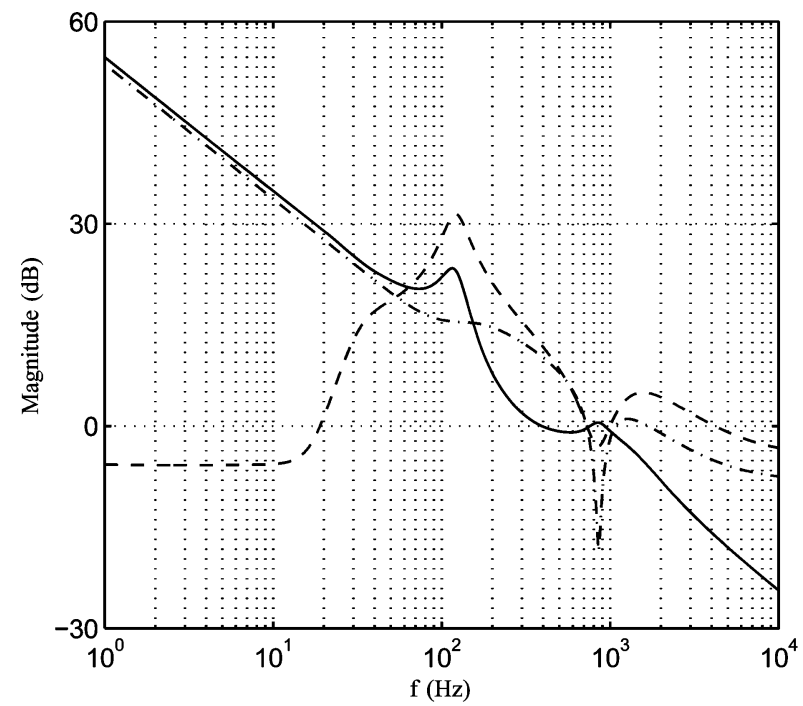

Fig. 10. Frequency response of the designed controllers $K_{c}(s)$ (solid), $K_{v}(s)($ dash$)$, and $\tilde{K}_{c}(s)$ (dash dot).

controllers conform to the design requirements. In particular, $K_{c}(s)$ is a high-gain controller at low frequencies within the bandwidth afforded by the capacitive sensor. On the other hand, $K_{v}(s)$ maintains a low gain within the bandwidth of the capacitive sensor, but applies a high gain beyond $20 \mathrm{~Hz}$. This "frequency sharing" enables the two controllers to maintain satisfactory tracking of the reference signal over the bandwidth of interest.

The final stage of the controller synthesis involves designing the feedforward controller $K_{\mathrm{fd}}(s)$ to shape the reference signal based on the achieved closed-loop frequency response $T_{y_{x} r}(s)$. The feedforward controller should be chosen such that

$$
K_{\mathrm{fd}}(s) \approx T_{y_{x} r}^{-1}(s)
$$

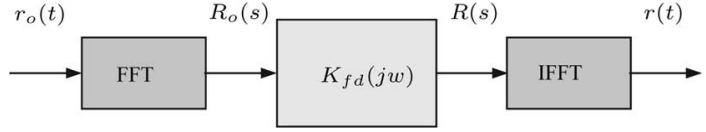

Fig. 11. Procedure to obtain shaped reference $r(t)$.

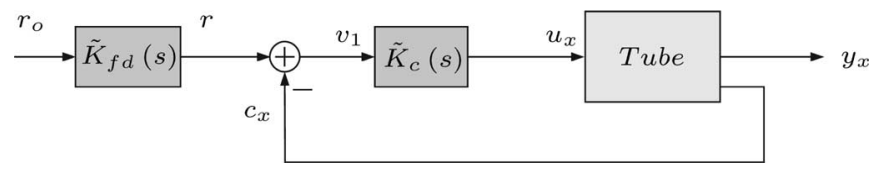

Fig. 12. 2-DOF control block diagram for the one-sensor-based $H_{\infty}$ controller.

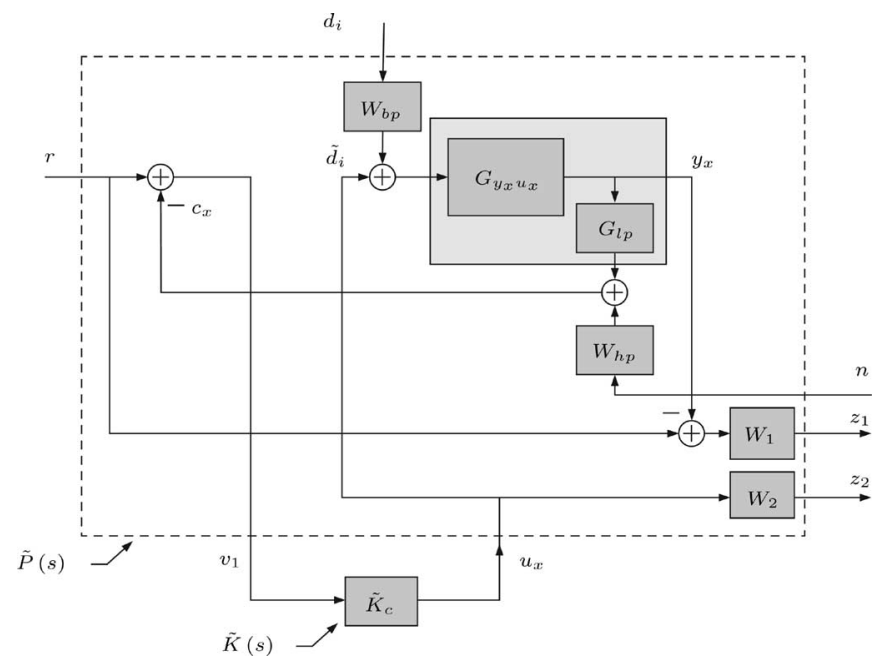

Fig. 13. Feedback control block diagram with weighting functions for the one-sensor-based $H_{\infty}$ controller.

Since the reference signal $r_{o}(t)$ is known and the frequency $T_{y_{x} r}(j w)$ can be measured in advance, the shaped reference signal $r(t)$ can be obtained offline, as shown in Fig. 11. This inversion is generally done over the frequency range for which a satisfactory model of the closed-loop system is available, in this case, up to $1600 \mathrm{~Hz}$.

\section{B. One-Sensor-Based $H_{\infty}$ Controller}

The purpose of this section is to demonstrate the immediate benefit of using the strain voltage sensor in addition to the low-bandwidth capacitive sensor. The controller designed here utilizes only the low-bandwidth capacitive sensor to obtain the tip displacement measurement for feedback. To make a fair comparison with the two-sensor-based $H_{\infty}$ controller, a 2-DOF controller, with a structure depicted in Fig. 12, was designed and implemented. Similar weighting functions (7)-(10) were used in synthesizing the $H_{\infty}$ controller $\tilde{K}_{c}(s)$. The feedback control block diagram with the weighting functions is illustrated in Fig. 13. A feedforward controller $\tilde{K}_{\mathrm{fd}}(s)$ was also designed and implemented in a similar manner as detailed in the previous section.

The achieved sensitivity function $\tilde{S}(s)$ for this control scheme is shown in Fig. 9. It can be observed that $\tilde{S}(s)$ does not match the desired sensitivity function $W_{1}^{-1}(s)$. This is a clear 


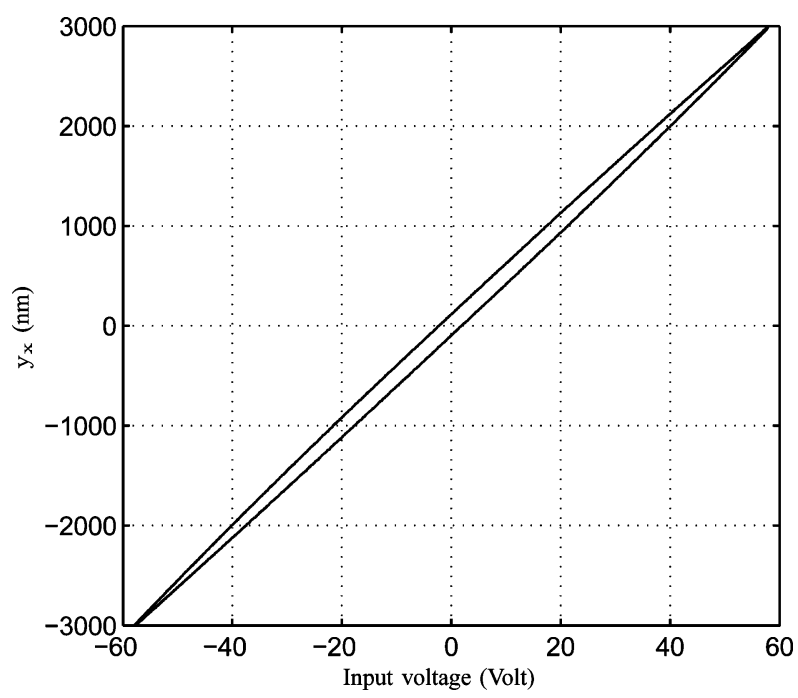

Fig. 14. Hysteresis plot of open-loop 5-Hz scan using voltage amplifier.

indication that a controller designed with one sensor alone is not capable of satisfying the design goals articulated before.

Fig. 10 plots the frequency response of the feedback controller $\tilde{K}_{c}(s)$. This is rather similar to $K_{c}(s)$ with the clear exception that it includes a notch filter at the first resonance frequency of the tube.

\section{RESULTS}

This section presents experimental results obtained from the two control schemes proposed in this paper. In order to measure the true deflection $y_{x}$, a capacitive sensor with a bandwidth of $10 \mathrm{kHz}$ was used in all tests. The sensor's signal was passed through a second-order Butterworth low-pass filter with a cutoff frequency of $100 \mathrm{~Hz}$. This latter signal was made available to the feedback controllers $K_{c}(s)$ and $\tilde{K}_{c}(s)$. Note that this low-pass filter is implemented simultaneously with the feedback controllers using the same real-time rapid prototyping system. Therefore, it should be considered as an integral part of $K_{c}(s)$ and $\tilde{K}_{c}(s)$. Inclusion of a low-pass filter in this arrangement is not entirely necessary since $K_{c}(s)$ and $\tilde{K}_{c}(s)$ are designed to operate at low bandwidths. This filter is incorporated in the design to emphasize the fact that this methodology works fine even with a low-bandwidth (and thus inexpensive) displacement sensor.

\section{A. Hysteresis Reduction}

The presence of hysteresis in the prototype nanoposiotioner was investigated by applying a $5-\mathrm{Hz}$ sinusoidal signal to the piezoelectric tube and measuring its deflection in open loop. A single-tone low-frequency signal was chosen here in order to avoid excitation of the first resonant mode of the tube. Also, this ensures that only the nonlinear component of the deflection is captured, since at such a low frequency, the linear dynamics of the tube resembles a simple gain with hardly any phase shift.

The tube was made to deflect large distance $( \pm 3.0 \mu \mathrm{m})$ so that the presence of hysteresis could be clearly observed. The effects of hysteresis were evaluated when the tube was driven by: 1) a

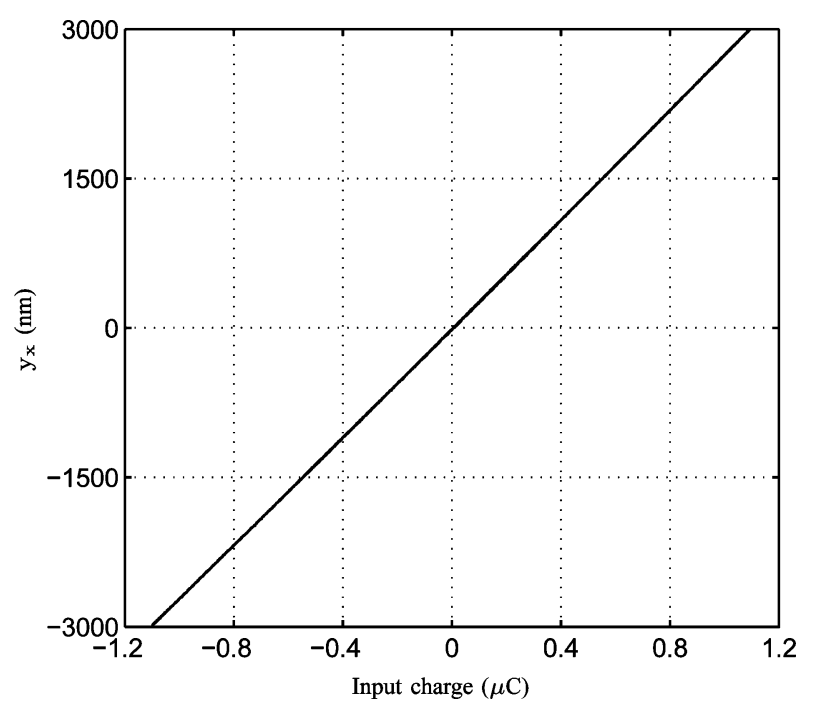

Fig. 15. Hysteresis plot of open-loop 5-Hz scan using charge amplifier

TABLE I

NUMERICAL QUANTIFICATION OF HYSTERESIS

\begin{tabular}{|c|c|c|}
\hline Configurations & $\begin{array}{c}\text { Max. output } \\
\text { hysteresis }\end{array}$ & $\begin{array}{c}\text { Max. input } \\
\text { hysteresis }\end{array}$ \\
\hline Voltage amp. & $207.50 \mathrm{~nm}(3.5 \%)$ & 4.12 volt $(3.6 \%)$ \\
\hline Charge amp. & $11.60 \mathrm{~nm}(0.19 \%)$ & $4.60 \mathrm{nC}(0.21 \%)$ \\
\hline
\end{tabular}

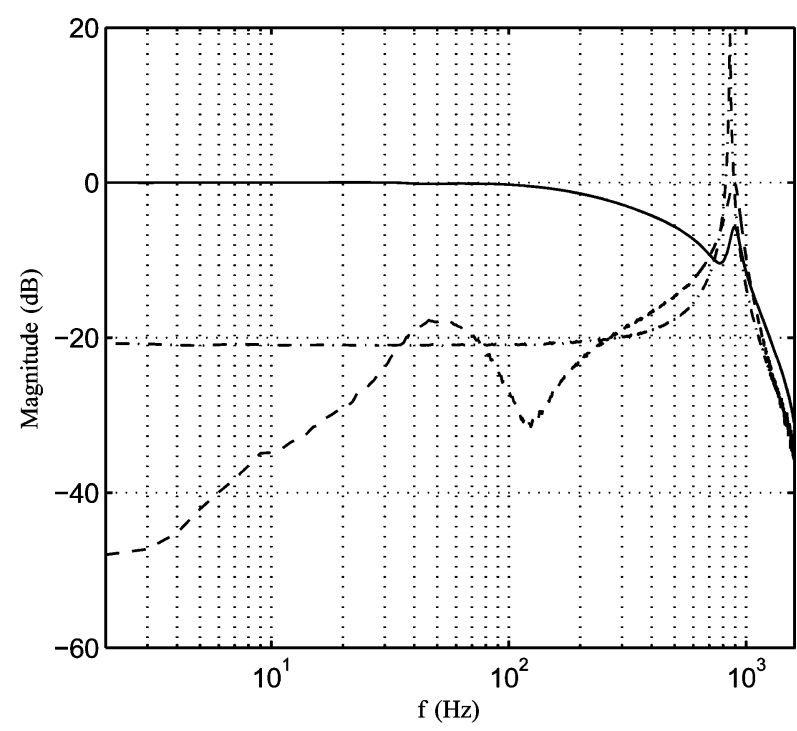

Fig. 16. Experimentally obtained frequency responses of $T_{y_{x} r}(i \omega)$ (solid), $T_{y_{x} \tilde{d}_{i}}(i \omega)$ (dash), and $G_{y_{x} u_{x}}(i \omega)$ (dash dots).

voltage amplifier and 2) a charge amplifier. For each case, the corresponding input signal and the tip deflection were recorded. Figs. 14 and 15 illustrate the plots of tip deflection versus input signal for voltage and charge, respectively. A clear reduction of hysteresis can be observed when the tube is driven by the charge amplifier. In order to quantify the improvement, the presence of hysteresis was measured in terms of the maximum (input and output) percentage deflection from a straight line [34]. The results are tabulated in Table I and clearly demonstrate the immediate benefit of driving the tube with a charge amplifier. 


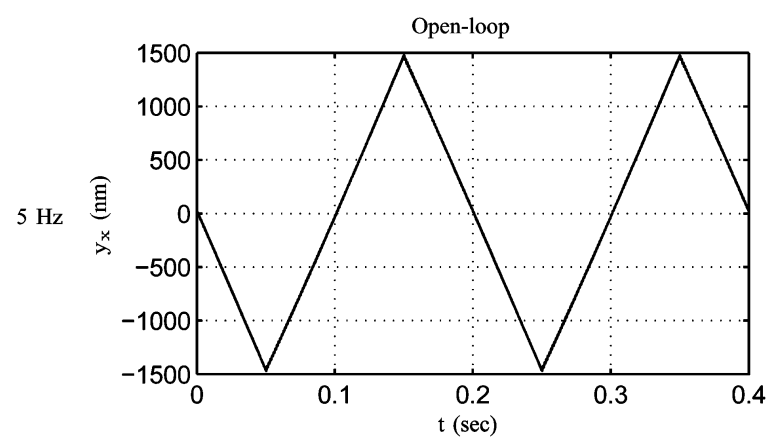

(a)

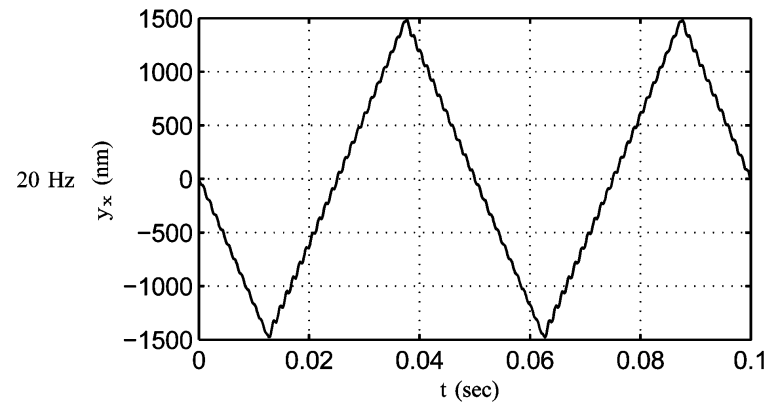

(b)

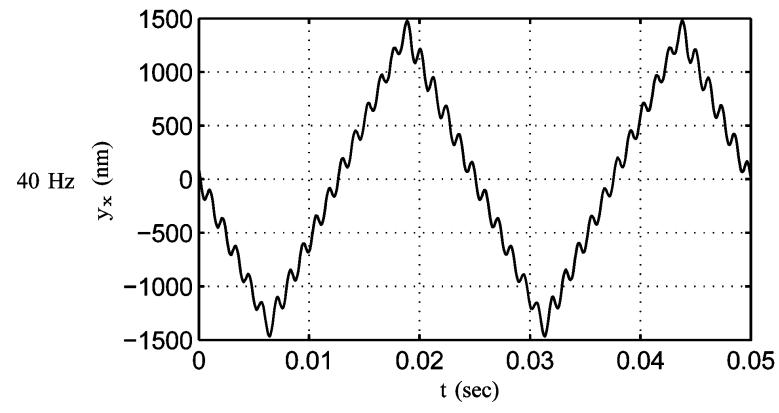

(c)

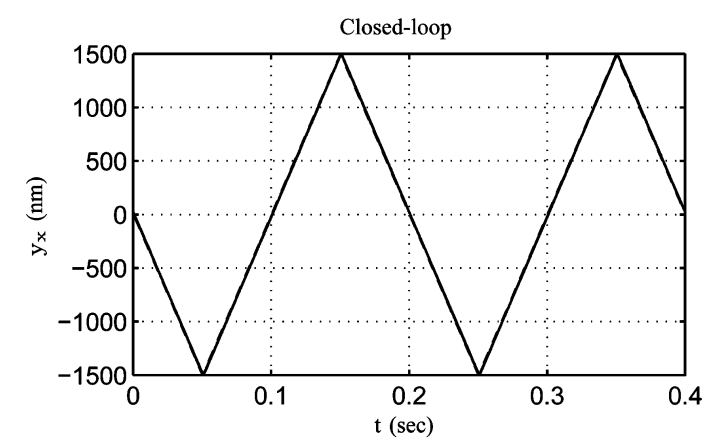

(d)

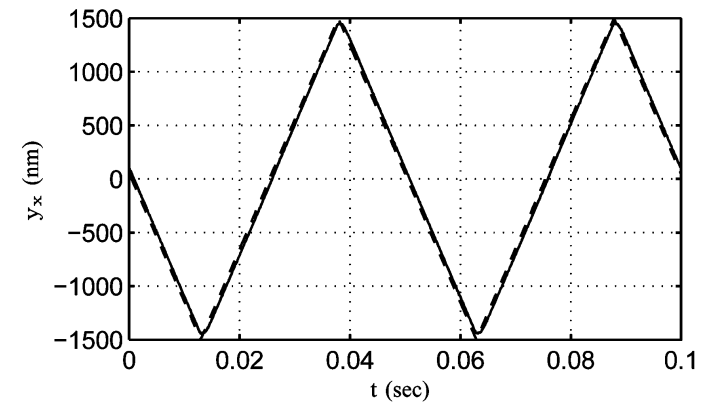

(e)

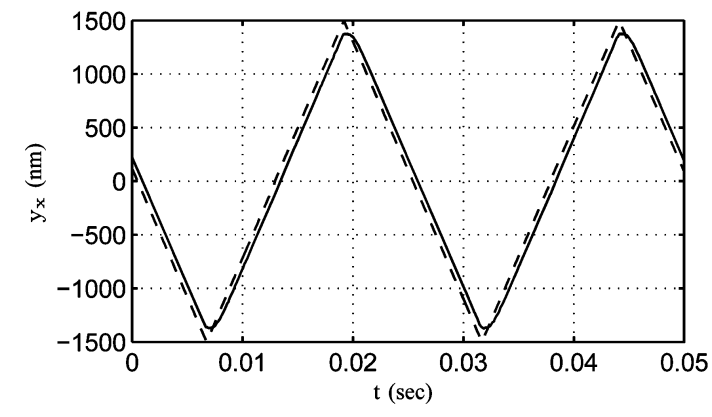

(f)

Fig. 17. Open-loop (left) and closed-loop (right) time response plots of 5-, 20-, and 40-Hz scan.

Although the hysteresis is not reduced to an absolute zero, it is made so small that the actuator can effectively be considered a linear device.

\section{B. Closed-Loop Frequency Response}

The performance of the feedback two-sensor-based controller was first evaluated by measuring the closed-loop responses of the nanopositioner using the spectrum analyzer. In Fig. 16, the closed-loop frequency responses $T_{y_{x} r}(i \omega)$ and $T_{y_{x} \tilde{d}_{i}}(i \omega)$ are plotted along with the open-loop frequency response $G_{y_{x} u_{x}}(i \omega)$. By inspecting the frequency response of $T_{y_{x} r}(i \omega)$, we conclude that the closed-loop system has a bandwidth of $310 \mathrm{~Hz}$. Also, a damping of $20 \mathrm{~dB}$ at the first resonant mode is evident from the frequency response of $T_{y_{x} \tilde{d}_{i}}(i \omega)$. Note that, the frequency response also shows that the closedloop system is insensitive to low-frequency input disturbances. Hence, the closed-loop system will perform in a satisfactory manner in the presence of low-frequency vibrations and disturbances. Overall, the two-sensor-based controller satisfies all the performance criteria.

\section{Time Response}

The ultimate purpose of the $x$-axis control loop is to allow for satisfactory tracking of a fast triangular set point. This is necessary if the actuator is to be used for fast scanning probe microscopy. First column of Fig. 17 plots the open-loop time responses of the nanopositioner due to 5-, 20-, and 40- $\mathrm{Hz}$ triangular input signals. It can be observed that as the frequency of the input signal increases, the extent to which the nanopositioner's motion becomes affected by the induced vibrations also increases. Particularly at $40 \mathrm{~Hz}$, the nanopositioner's motion is badly affected by amplification of the 11th harmonic $(840 \mathrm{~Hz})$ of the triangular signal that is close to the first resonance frequency of the piezoelectric tube (about $850 \mathrm{~Hz}$ ).

The right-hand column of Fig. 17 [Fig. 17(d)-(f)] compares the closed-loop response of the nanopositioner under the two-sensor-based $H_{\infty}$ controller with the desired set point. It can be observed that the controller successfully damps the induced vibrations and provides excellent tracking performance, particularly at low frequencies. The damping of $20 \mathrm{~dB}$ at the first resonant mode of the nanopositioner is sufficient to avoid the amplification of the harmonics near the first resonance fre- 
TABLE II

RMS VALUES OF TRACKING ERROR

\begin{tabular}{|c|c|c|c|}
\hline Scan Freq. & \multirow{2}{*}{ Open-loop } & \multicolumn{2}{|c|}{ Closed-loop } \\
\cline { 3 - 4 } & & Two-sensor & One-sensor \\
\hline $5 \mathrm{~Hz}$ & $12.2 \mathrm{~nm}$ & $1.9 \mathrm{~nm}$ & $2.0 \mathrm{~nm}$ \\
\hline $10 \mathrm{~Hz}$ & $9.9 \mathrm{~nm}$ & $2.3 \mathrm{~nm}$ & $2.4 \mathrm{~nm}$ \\
\hline $20 \mathrm{~Hz}$ & $10.7 \mathrm{~nm}$ & $3.2 \mathrm{~nm}$ & $3.4 \mathrm{~nm}$ \\
\hline $30 \mathrm{~Hz}$ & $17.5 \mathrm{~nm}$ & $3.1 \mathrm{~nm}$ & $9.1 \mathrm{~nm}$ \\
\hline $40 \mathrm{~Hz}$ & $67.0 \mathrm{~nm}$ & $7.6 \mathrm{~nm}$ & $15.6 \mathrm{~nm}$ \\
\hline
\end{tabular}

quency. Note that the controller's ability to track the reference signal at its corners is reduced when the frequency of the input signal is increased. This effect is clearly visible in Fig. 17(f) and is mainly due to the limited closed-loop bandwidth of the system. The feedforward controller incorporated into the tracking system works to correct this effect. However, its effectiveness is limited by the accuracy of the closed-loop model used in the inversion. Nevertheless, this should not be viewed as a drawback as it is common practice in SPMs to limit the image size to within a certain percentage of the available window with the understanding that, quite often, the image could be distorted around the edges.

Open-loop and closed-loop tracking errors for various scan frequencies are tabulated in Table II. The tracking errors are determined by calculating the rms difference between the measured displacement and the reference signal for $90 \%$ of the scan range (ignoring the top and bottom 5\% of the scans). A fixed phase shift between the measured displacement and the reference input can be observed in Fig. 17(d)-(f). In calculating the tracking errors, these phase shifts were removed. At the slow speed scan of $5 \mathrm{~Hz}$, the controller displays excellent tracking performance with tracking error of only $1.9 \mathrm{~nm}$, i.e., $0.06 \%$ of the entire scan range. The tracking error remains satisfactory even as the scan frequency is increased as high as $40 \mathrm{~Hz}$, as shown in Table II. The error does not exceed $0.25 \%$ of the scan range. Note that in measuring the displacements, a highbandwidth capacitive sensor with a bandwidth of $10 \mathrm{kHz}$ was used. At this bandwidth, the rms noise level of the sensor is calculated to be $1.75 \mathrm{~nm}$. The stochastic noise affecting the "true" output of the nanopositioner is significantly lower than this, since the said noise is largely due to the capacitive sensor signal that is low-pass filtered at $100 \mathrm{~Hz}$. The stochastic noise arising from the strain voltage signal can effectively be ignored in this case due to its extremely low noise density.

\section{One-Sensor-Based $H_{\infty}$ Controller}

For the sake of completeness, closed-loop performance of the nanopositioner with the one-sensor-based $H_{\infty}$ controller is studied here. Fig. 18 illustrates closed-loop performance of this controller. It can be observed that the bandwidth of the closedloop system is about a third of that of the two-sensor-based system. By comparing $T_{y_{x} \tilde{d}_{i}}(i \omega)$ and $G_{y_{x} u_{x}}(i \omega)$ in Fig. 18, it can be concluded that $\tilde{K}_{c}(s)$ does not damp the first resonance mode of the tube in a satisfactory manner. This is of little surprise since this mode is out of the $100-\mathrm{Hz}$ bandwidth of the controller. However, no induced vibrations are observed when the tube is made to track various triangular waveforms, as illustrated in

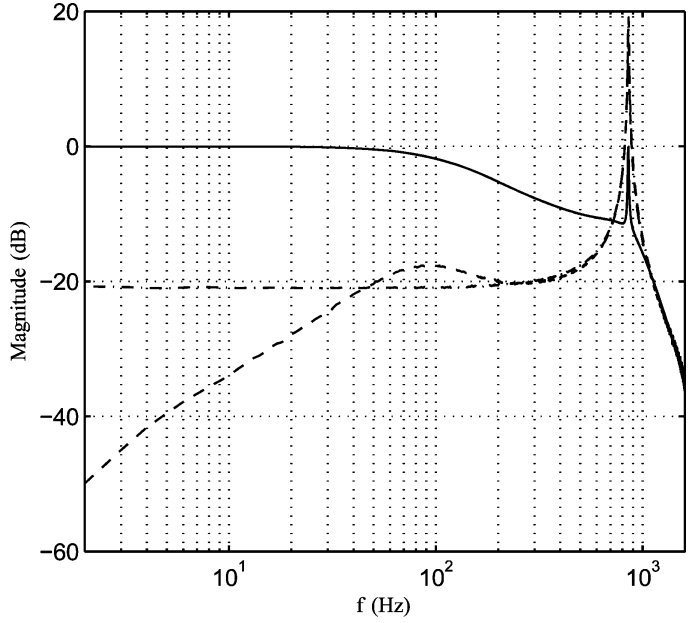

Fig. 18. Experimentally obtained closed-loop frequency responses using one-sensor-based $H_{\infty}$ controller, $T_{y_{x} r}(i \omega)$ (solid), $T_{y_{x} d_{i}}(i \omega)$ (dash), and $G_{y_{x} u_{x}}(i \omega)$ (dash dots).

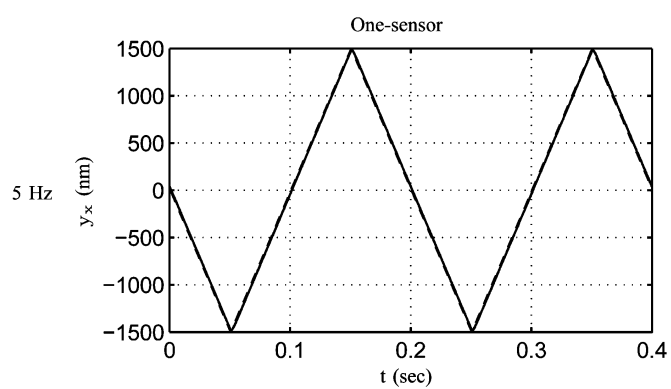

(a)

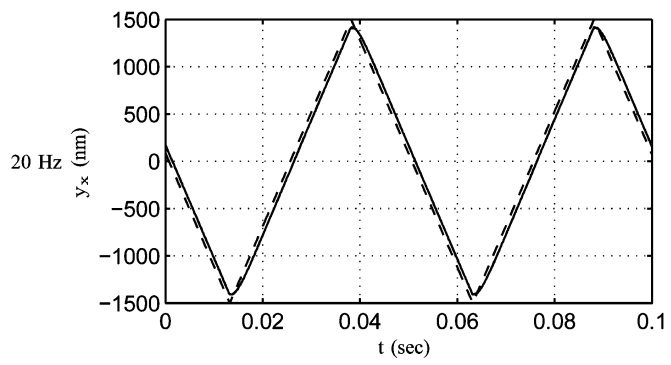

(b)

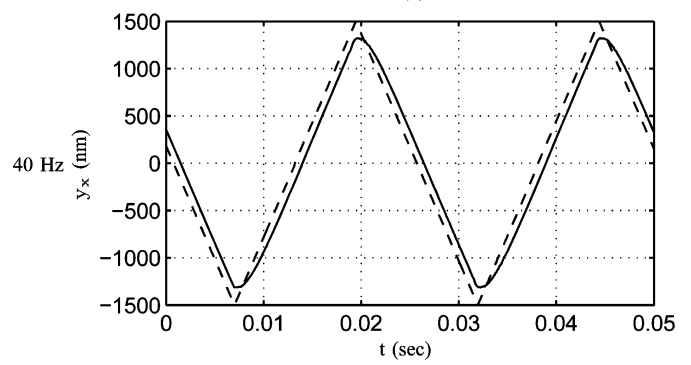

(c)

Fig. 19. Closed-loop time response plots of 5-, 20-, and 40-Hz scan using one-sensor-based $H_{\infty}$ controller.

Fig. 19. This is due to the existence of the notch filter in $\tilde{K}_{c}(s)$. Also, the two-sensor-based controller performs better in terms of rejecting low-frequency vibrations and noise.

The closed-loop tracking errors due to $\tilde{K}_{c}(s)$ are tabulated in the last column of Table II. It can be seen that up to $20-\mathrm{Hz}$ 
scanning frequency, the tracking performance is comparable to that obtained through $K_{c}(s)$. However, at higher scan frequencies, the tracking performance decrease rapidly, as illustrated in Table II and Fig. 19.

\section{CONCLUSION}

We described how a high-bandwidth low-noise two-sensorbased controller could be designed for a piezoelectric tube nanopositioner. The two-input one-output controller uses measurements obtained from a capacitive displacement sensor at low frequencies, and the piezoelectric voltage signal at high frequencies. By keeping the capacitive sensor loop bandwidth low, the effect of sensor noise on the overall system is significantly reduced. Having access to the piezoelectric voltage signal allows the controller to achieve tracking over a wide bandwidth and successful damping of the resonant mode of the scanner by about $20 \mathrm{~dB}$. Overall, this paper provides a further justification for using complementary sensors, whenever possible, in nanoscale positioning systems in line with results reported in [31] and [35].

\section{REFERENCES}

[1] T. Bailey and J. E. Hubbard, "Distributed piezoelectric-polymer active vibration control of a cantilever beam," J. Guid., Control, Dyn., vol. 8, no. 5, pp. 605-611, 1985.

[2] C. R. Fuller, S. J. Elliot, and P. A. Nelson, Active Control of Vibration. San Francisco, CA: Academic, 1996.

[3] S. O. R. Moheimani and A. J. Fleming, Piezoelectric Transducers for Vibration Control and Damping. Germany: Springer-Verlag, 2006, ISBN: 1-84628-331-0.

[4] N. W. Hagood and A. von Flotow, "Damping of structural vibrations with piezoelectric materials and passive electrical networks," J. Sound Vibration, vol. 146, no. 2, pp. 243-268, 1991.

[5] D. Halim and S. O. R. Moheimani, "Experimental implementation of spatial $H_{\infty}$ control on a piezoelectric laminate beam," IEEE/ASME Trans. Mechatronics, vol. 7, no. 3, pp. 346-356, Sep. 2002.

[6] I. R. Petersen and H. R. Pota, "Minimax LQG optimal control of a flexible beam," Control Eng. Practice, vol. 11, no. 11, pp. 1273-1287, Nov. 2003.

[7] W. H. Shields, J. Ro, and A. M. Baz, "Control of sound radiation from a plate into an acoustic cavity using active piezoelectric-damping composites," in Proc. SPIE Smart Struct. Mater.: Math. Control Smart Struct., 1997 , pp. $70-90$

[8] C. H. Hansen and S. D. Snyder, Active Control of Noise and Vibration. London, U.K.: E \& FN Spon, 1997.

[9] L. Bor-Shun, H. Jyun-Jhang, W. Wen-Jong, and S. Wen-Pin, "Mems power receiver using piezoelectric cantilever beams and interdigitated electrodes," in Proc. IEEE Int. Conf. Syst., Man, Cybern., 2006, vol. 4, pp. 3227-3232.

[10] E. Meyer, H. J. Hug, and R. Bennewitz, Scanning Probe Microscopy: The Lab on a Tip. Berlin, Germany: Spring-Verlag, 2004.

[11] G. Binnig and D. P. E. Smith, "Single-tube three-dimensional scanner for scanning tunneling microscopy," Rev. Sci. Instrum., vol. 57, no. 8, pp. 1688-1689, Aug. 1986

[12] V. L. Mironov, Fundamentals of Scanning Probe Microscopy. Moscow: Nizhniy Novgorod, 2004

[13] D. Croft, G. Shed, and S. Devasia, "Creep, hysteresis, and vibration compensation for piezoactuators: Atomic force microscopy application," $J$ Dyn. Syst., Meas., Control, vol. 123, pp. 35-43, 2001.

[14] N. Tamer and M. Dahleh, "Feedback control of piezoelectric tube scanners," in Proc. 33rd Conf. Decis. Control, 1994, pp. 1826-1830.

[15] A. Daniele, S. Salapaka, M. V. Salapaka, and M. Dahleh, "Piezoelectric scanners for atomic force microscopes: Design of lateral sensors, identification and control," in Proc. Am. Control Conf., 1999, pp. 253-257.

[16] O. M. E. Rifai and K. Youcef-Tomi, "Coupling in piezoelectric tube scanners used in scanning probe microscopes," in Proc. Am. Control Conf., Arlington, VA, 2001, pp. 3251-3255.
[17] G. Schitter and A. Stemmer, "Identification and open-loop tracking control of a piezoelectric tube scanner for high-speed scanning-probe microscopy," IEEE Trans. Control Syst. Technol., vol. 12, no. 3, pp. 449454, May 2004.

[18] G. Schitter, P. Menold, H. F. Knapp, F. Allgower, and A. Stemmer, "High performance feedback for fast scanning atomic force microscopes," Rev. Sci. Instrum., vol. 72, no. 8, pp. 3320-3327, 2001

[19] S. Devasia, E. Eleftheriou, and S. O. R. Moheimani, "A survey of control issues in nanopositioning," IEEE Trans. Control Syst. Technol., vol. 15, no. 5 , pp. 802-823, Sep. 2007.

[20] R. C. Barrett and C. F. Quate, "Optical scan-correction system applied to atomic force microscopy," Rev. Sci. Instrum., vol. 62, no. 6, pp. 1393 1399, Jun. 1991.

[21] K. Leang and S. Devasia, "Hysteresis, creep, and vibration compensation for piezoactuators: Feedback and feedforward control," in Proc. 2nd IFAC Conf. Mechatronic Syst., Berkeley, CA, Dec. 2002, pp. 283-289.

[22] R. Comstock, "Charge control of piezoelectric actuators to reduce hysteresis effect," U.S. Patent 4263 527, 1981.

[23] C. Newcomb and I. Flinn, "Improving the linearity of piezoelectric actuators using charge feedback," Electron. Lett., vol. 18, no. 11, pp. 442-444, May 1982.

[24] A. J. Fleming and S. O. R. Moheimani, "Sensorless vibration suppression and scan compensation for piezoelectric tube nanopositioners," IEEE Trans. Control Syst. Technol., vol. 14, no. 1, pp. 33-44, Jan. 2006.

[25] A. J. Fleming and S. O. R. Moheimani, "A grounded load charge amplifier for reducing hysteresis in piezoelectric tube scanners," Rev. Sci. Instrum., vol. 76, no. 7, pp. 073707-1-073707-5, 2005.

[26] B. J. G. Vautier and S. O. R. Moheimani, "Charge-driven piezoelectric actuators for structural vibration control: Issues and implementation," Smart Mater. Struct., vol. 14, no. 4, pp. 575-586, 2005.

[27] G. Schitter and A. Stemmer, "Identification and open-loop tracking control of a piezoelectric tube scanner for high-speed scanning-probe microscopy," IEEE Trans. Control Syst. Technol., vol. 12, no. 3, pp. 449-454, May 2004.

[28] B. Bhikkaji, M. Ratnam, A. J. Fleming, and S. O. R. Moheimani, "Highperformance control of piezoelectric tube scanners," IEEE Trans. Control Syst. Technol., vol. 5, no. 5, pp. 853-866, Sep. 2007.

[29] B. Bhikkaji, M. Ratnam, and S. O. R. Moheimani, "PVPF control of piezoelectric tube scanners," Sens. Actuators, A. Phys., vol. 135, no. 2, pp. 700-712, Apr. 2007.

[30] A. J. Fleming, A. Wills, and S. O. R. Moheimani, "Sensor fusion for improved control of piezoelectric tube scanners," IEEE Trans. Control Syst. Technol., vol. 16, no. 6, pp. 1265-1276, Nov. 2008.

[31] A. J. Fleming, A. Wills, and S. O. R. Moheimani, "Sensor fusion for improved control of piezoelectric tube scanners," in Proc. IEEE/ASME Int. Conf. Adv. Intell. Mechatronics, Zurich, Switzerland, Sep. 4-7, 2007, pp. 1-6.

[32] T. McKelvey, H. Ackay, and L. Ljung, "Subspace-based identification of infinite-dimensional multi-variable systems from frequency-response data," IEEE Trans. Autom. Control., vol. AC-41, no. 7, pp. 960-979, Jul. 1996.

[33] T. McKelvey, A. J. Fleming, and S. O. R. Moheimani, "Subspace-based system identification for an acoustic enclosure," ASME J. Vib. Acoust., vol. 124, no. 3, pp. 414-419, Jul. 2002.

[34] S. Salapaka, A. Sebastian, J. P. Cleveland, and M. V. Salapaka, "High bandwidth nanopositioner: A robust control approach," Rev. Sci. Instrum., vol. 73, no. 9, pp. 3232-3241, 2002.

[35] A. Pantazi, A. Sebastian, H. Pozidis, and E. Eleftheriou, "Two-sensorbased $H_{\infty}$ control for nanopositioning in probe storage," in Proc. IEEE Conf. Decision Control, Dec. 12-15, 2005, pp. 1174-1179.

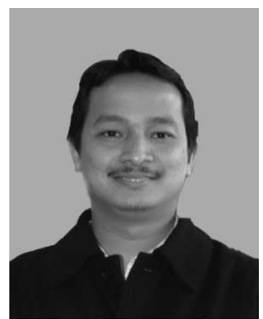

Iskandar A. Mahmood was born in Malaysia in 1975. He received the B.Eng. and M.Sc. degrees in mechatronics engineering from the International Islamic University Malaysia, Kuala Lumpur, Malaysia, in 1999 and 2004, respectively. He is currently working toward the Ph.D. degree in electrical engineering at the University of Newcastle, Callaghan, N.S.W., Australia.

Since 2005 , he has been with the Laboratory of Dynamics and Control of Nanosystems, University of Newcastle. 


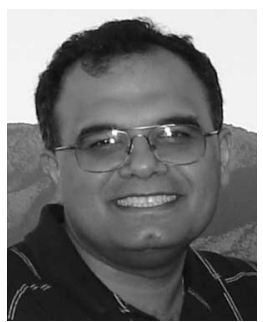

S. O. Reza Moheimani (SM'00) received the Ph.D. degree in electrical engineering from the University of New South Wales at the Australian Defence Force Academy, Canberra, A.C.T., Australia, in 1996.

$\mathrm{He}$ is currently a Professor in the School of Electrical Engineering and Computer Science, University of Newcastle, Callaghan, N.S.W., Australia, where he is also the Assistant Dean Research for the Faculty of Engineering and Built Environment. He is the Associate Director of the Australian Research Council (ARC) Centre for Complex Dynamic Systems and Control, an Australian Government Centre of Excellence. He has held several visiting appointments at IBM Zurich Research Laboratory, Switzerland. He has published two books, several edited volumes, and more than 150 refereed articles in archival journals and conference proceedings. His current research interests include applications of control and estimation in nanoscale positioning systems for scanning probe microscopy, and control of electrostatic microactuators in microelectromechanical systems (MEMS) and data storage systems.

Prof. Moheimani is a Fellow of the Institute of Physics, U.K. He is the recipient of the 2007 IEEE TRANSACTIONS ON CONTROL SYSTEMS TECHNOLOGY Outstanding Paper Award. He has served on the Editorial Board of a number of journals including the IEEE TRANSACTIONS ON CONTROL SYSTEMS TECHNOLOGY, and chaired several international conferences and workshops.

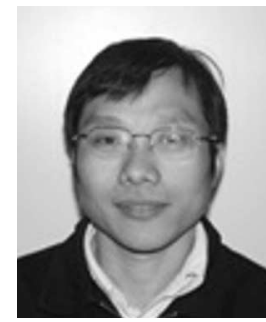

Kexiu Liu received the Ph.D. degree in electrical engineering from the National University of Singapore, Singapore, in 2001.

He was with Seagate Hard Disk Research Center, Singapore, for six years. He is currently a Research Academic in the School of Electrical Engineering and Computer Science, University of Newcastle, Callaghan, N.S.W., Australia. His current research interests include optimal control, adaptive control, model predictive control, and hard disk application. 